\title{
Deep vein thrombosis and pulmonary embolism in a patient with acute type $B$ aortic dissection: a case report
}

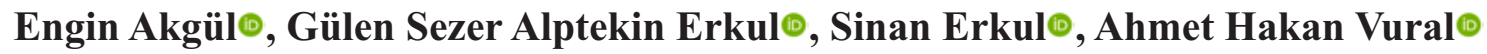 \\ Department of Cardiovascular Surgery, Dumlupınar University, Evliya Çelebi Training and Research Hospital, Kütahya, Turkey
}

DOI: $10.18621 /$ eurj.403641

\begin{abstract}
Acute dissection involving ascending aorta contains high risk of mortality and requires surgical treatment immediately. Venous thrombosis can manifested as deep vein thrombosis or pulmonary embolism. It may be isolated or complication of another disease. Because of pulmonary thromboembolism risk, treatment of deep vein thrombosis is strongly recommended. A 61-year-old male patient with severe back pain and shortness of breath presented to the emergency service. The findings of the physical examinations, chest $\mathrm{x}$-ray and electrocardiogram were normal. Contrast-enhanced computerized tomography showed an aortic intimal tear that started just below the subclavian artery and extended into the iliac arteries. The patient was hospitalized and the medical treatment started. On the $4^{\text {th }}$ day of clinical follow-up, pain and swelling started at his right leg with severe shortness of breath. Venous Doppler ultrasound was performed and there were thrombosis at popliteal, femoral and even at iliac veins. Computed tomography showed pulmonary embolism at pulmonary trunk. Aortic dissection treated with endovascular stent graft firstly to prevent aortic rupture because of anticoagulation and then pulmonary embolism treated with anticoagulant drugs. Hypercoagulation is a self defence of the body for limiting the aortic intimal tear to prevent aortic rupture. So many complications could be seen because of this situation and the physicians should be awaken for this.
\end{abstract}

Keywords: acute aortic dissection, deep vein thrombosis, pulmonary embolism, endovascular stent graft, anticoagulation

Received: March 9, 2018; Accepted: May 2, 2018; Published Online: June 26, 2018

A ortic dissection is classified according to the location of the tear [1]. Stanford type A aortic dissection has a high mortality rate and should be promptly directed to surgery whereas Stanford type B aortic dissection can be followed without surgery [2, 3]. However, organ malperfusion may be likely if the dissection flap or intramural hematoma extends distally involving visseral and iliac arteries in type B aortic dissection [4]. On the other hand deep vein thrombosis (DVT) and pulmonary thromboembolism are some of the manifestations of venous thromboembolism (VTE) $[5,6]$. Herein we aimed to discuss a case of acute type $\mathrm{B}$ aortic dissection with VTE possibly provoked by the dissection itself or our treatment approach.

\section{CASE PRESENTATION}

A 61-year-old-male patient presented with complaints such as severe back pain that begins two hours before admission. He had a history of

Address for correspondence: Engin Akgül, MD., Dumlupınar University, Evliya Çelebi Training and Research Hospital, Department of Cardiovascular Surgery, Kütahya, Turkey 
hypertension for 10 years. The arterial blood pressure measured 190/130 mm Hg bilaterally. The other findings of physical examination were ordinary as were also electrocardiographic findings and chest $\mathrm{x}$ ray. Contrast-enhanced computerized tomographic angiography (CTA) was performed. It showed an extensive dissection of the aorta arising distal to the left subclavian artery and extending into the common iliac arteries bilaterally. Antihypertensive, analgesic and sedative therapies arranged to stabilize the blood pressure. The pain regressed and the blood pressure controlled with the medical therapy alone. The renal functions were normal and there was not an evidence of organ malperfusion thus surgical treatment was not planned. On the 4th day of clinical follow-up he had pain, swelling, erythema and an increase in the diameter of his right leg with severe shortness of breath although the arterial circulation of the lower extremities was intact with palpable arteries. Venous Doppler ultrasound imaging was performed and it

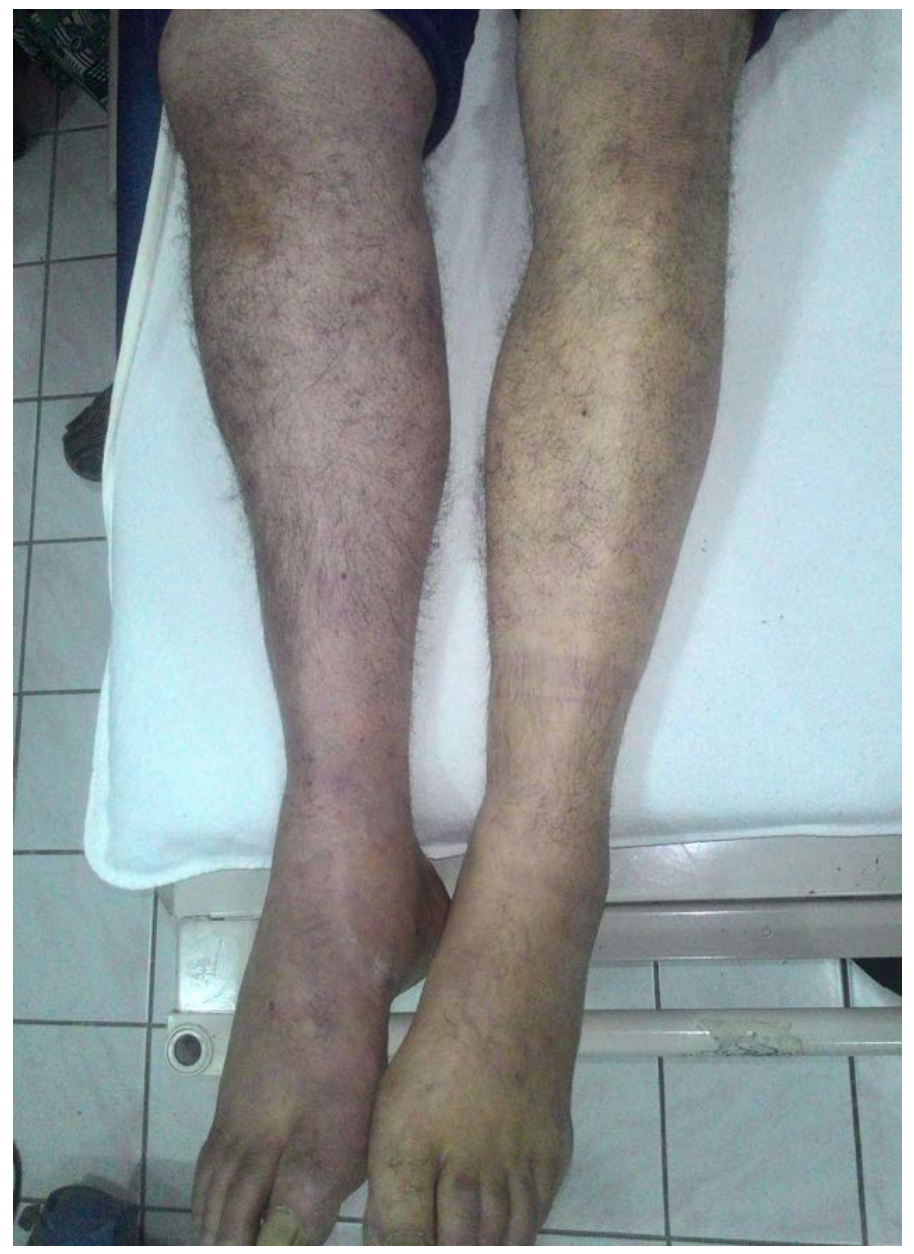

Figure 1. Edema of the right leg due to deep vein thrombosis. showed the acute thrombosis of the right popliteal, femoral and iliac veins. As the deep vein thrombosis was diagnosed, a CTA performed to evaluate pulmonary thromboembolism (Figure 1). It showed the segmental embolism in the main pulmonary trunk and in the branches of the bilateral pulmonary arteries (Figure 2). An effective dosage of anticoagulant therapy was not applied to avoid the rupture of the aorta. We decided to treat type B aortic dissection with endovascular stenting prominently to apply an adequate anticoagulant therapy soon afterwards. Thoracic endovascular aortic repair (TEVAR) was determined to perform (Figure 3). The left brachial artery used for the guidewire access for entering to the true lumen. Right femoral artery explored via a right femoral incision under local anesthesia. The wire moved forward into the descending aorta towards the right common femoral artery. A transvers arteriotomy made on the right common femoral artery to capture the guidewire with a clamp. Endovascular stent graft $(42 \times 42 \times 207 \mathrm{~mm}$, Medtronic, USA $)$ implanted in the descending aorta just below the left subclavian artery and above the fourth thoracal vertebral level. No complication occured. After TEVAR medical treatment for pulmonary thromboembolism and DVT revised. Anticoagulant treatment (Warfarin sodium) started the day after TEVAR for a target international normalized ratio (INR) level between 2-3. An effective dosage $(80 \mathrm{IU} / \mathrm{kg}$, enoxaparin sodium twice a day subcutaneously) of low molecular weight heparin (LMWH) applied from the day of the intervention until reaching the target INR. Shortness of breath and the edema of the leg regressed and the difference in diameter between two legs decreased after TEVAR in several days. The patient discharged on the $10^{\text {th }}$ day after TEVAR with an effective dosage regimen of warfarin sodium and acetylsalicylic acid treatment. The symptoms relieved at discharge. Three months after the discharge the patient had no symptom related to either VTE or the aortic dissection.

\section{DISCUSSION}

Type B aortic dissection may be accompanied with rupture, organ malperfusion, aortic aneurysmdilatation and severe pain [7]. Immediate surgery is one of the treatment options for complicated 


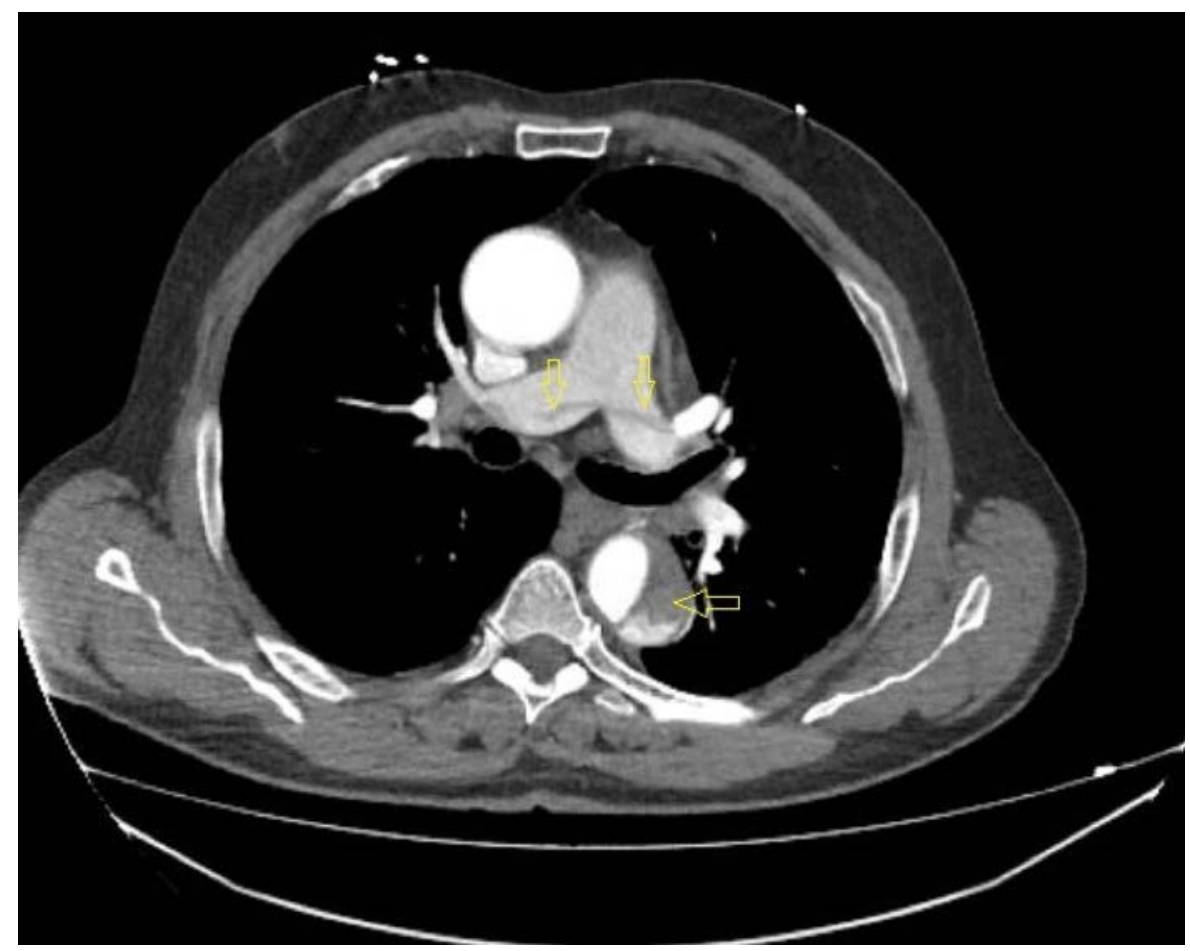

Figure 2. Thrombosis at bilateral pulmonary arteries and the aortic dissection flap were figured with vertical and horizontal arrows respectively.

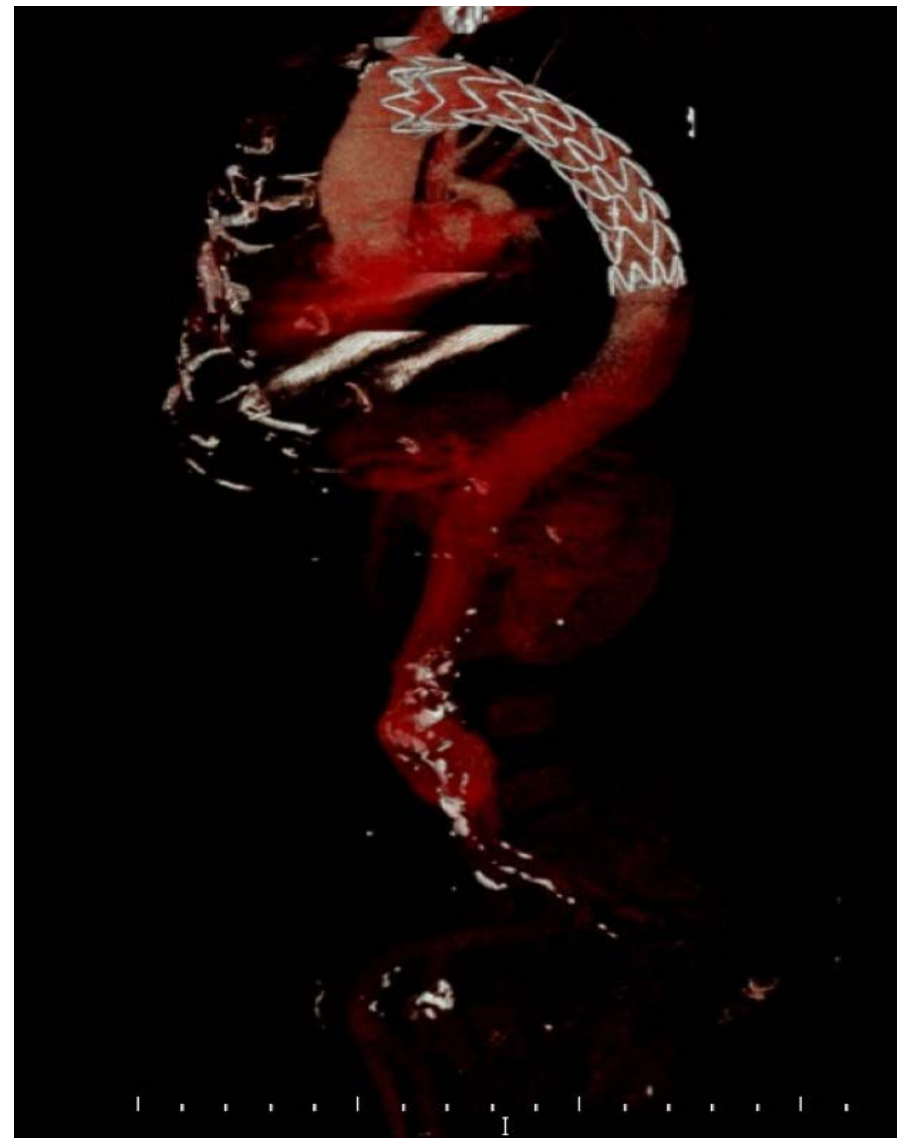

Figure 3. CT angiography image: the endovascular stent graft in the right position. dissections but many authors recommend TEVAR instead of open surgery as the initial treatment [8]. A conservative approach by pain medication and antihypertensive drugs is a more appropriate treatment option for the uncomplicated type B aortic dissection in many cases [9]. Even though the patients are determined as uncomplicated on admission, a significant majority of them develop severe complications and need further interventions on follow-up [9]. Because of these catastrophic complications, indications of TEVAR for type B aortic dissections should be expanded for some circumstances.

It was suggested that during aortic dissection hypercoagulability may be triggered as a self-defence and this may cause thromboembolism [10]. VTE is a miscellaneous entity in the presence of acute aortic dissection therefore the anticoagulant or the thrombolytic therapy essential for the treatment of VTE may cause recanalization of the false lumen of the aorta. At the meantime the tear may be expanded proximally or distally and the false lumen may turn into a devastating hematoma ending in a poor outcome for the patient [10]. On the other hand in a hypercoagulable circumstance, the pulmonary 
thromboembolism with or without DVT is a life threatening status which should be treated promptly. In our case thrombolytic therapy was contraindicated and the convenience for the application of LMWH was uncertain. The vena cava filter should be a treatment option to prevent pulmonary embolism in such cases [10]. However in our case acute pulmonary embolism was present from the time of the DVT diagnosis. Therefore we decided to treat the dissection site first by TEVAR and afterwards to apply an effective dosage of anticoagulant regimen. Three months after the discharge, the all symptoms of the patient were relieved. There are many studies which suggest the correlation of D-Dimer levels with redissection and VTE incidence among the patients with type B aortic dissection [11]. Although we couldn't find any recommendation for the treatment of VTE concomitant with aortic dissection in the literature considering it is a rare and an individual circumstance. However we think that it was life saving to treat aortic dissection prominently with an endovascular therapy option in our case.

\section{CONCLUSION}

Hypercoagulation is a self defence of the body for limiting the aortic intimal tear to prevent aortic rupture. Therefore the thromboembolism of the other vessels may be observed concomitantly. The patients should be evaluated individually and the endovascular treatment options should be kept in mind for such miscellaneous circumstances.

\section{Informed consent}

Written informed consent was obtained from the patient for publication of this case report and any accompanying images.

\section{Conflict of interest}

The authors declared that there are no potential conflicts of interest with respect to the research, authorship, and/or publication of this article.

\section{REFERENCES}

[1] Pape L, Awais M, Woznicki EM, Suzuki T, Trimarchi S, Evangelista A, et al. Presentation, diagnosis and outcomes of acute aortic dissection: 17-year trends from the International Registry of Acute Aortic Dissection. J Am Coll Cardiol 2015;66:350-8.

[2] Yavuz S. Alternative cannulation techniques in surgical repair for acute type A aortic dissection. Eur Res J 2016;2:1-6.

[3] Nienaber CA, Kische S, Rousseau H, Eggebrecht H, Rehders TC, Kundt G, et al. Endovascular repair of type B aortic dissection: longterm results of the randomized investigation of stent grafts in aortic dissection trial. Circ Cardiovasc Interv 2013;6:407-16.

[4] Grimm JC, Magruder JT, Crawford TC, Sciortino CM, Zehr KJ, Mandal K, et al. Differential outcomes of type A dissection with malperfusion according to affected organ system. Ann Cardiothorac Surg 2016;5:202-8.

[5] Tapson VF. Acute pulmonary embolism. N Engl J Med 2008;358:1037-52.

[6] Yavuz S, Toktas F, Goncu T, Eris C, Gucu A, Ay D, et al. Surgical embolectomy for acute massive pulmonary embolism. Int J Clin Exp Med 2014;7:5362-75.

[7] Appoo JJ, Bozinovski J, Chu MW, El-Hamamsy I, Forbes TL, Moon M, et al. Canadian Cardiovascular Society/Canadian Society of Cardiac Surgeons/Canadian Society for Vascular Surgery Joint Position Statement on Open and Endovascular Surgery for Thoracic Aortic Disease. Can J Cardiol 2016;32:703-13.

[8] Fattori R, Cao P, De Rango P, Czerny M, Evangelista A, Nienaber $\mathrm{C}$, et al. Interdisciplinary expert consensus document on management of type B aortic dissection. J Am Coll Cardiol 2013;61:1661-78.

[9] Sher A, Tadros RO. Is there a role for expanding the indication for TEVAR in acute type B aortic dissections? A further classification of uncomplicated dissections. J Vasc Endovasc Surg 2016;1:3.

[10] Kagawa Y, Ota S, Hoshino K, Yamada N, Nakamura M, Ito M. Acute pulmonary thromboembolism and deep vein thrombosis during the medical treatment of acute aortic dissection was successfully treated by the combination of inferior vena cava filter installation and anticoagulant therapy: a case report. Ann Vasc Dis 2015;8:36-9.

[11] Jo Y, Anzai T, Ueno K, Kaneko H, Kohno T, Sugano Y et al. Reelevation of $\mathrm{D}$-dimer as a predictor of re-dissection and venous thromboembolism after Stanford type B acute aortic dissection. Heart Vessels 2010;25:509-14. 